ARTICLE

\title{
Efficient DNA-assisted synthesis of trans-membrane gold nanowires
}

\author{
Maoxiang Guo ${ }^{1}$, Iván Hernández-Neuta ${ }^{2}$, Narayanan Madaboosi ${ }^{2}$, Mats Nilsson ${ }^{2}$ and Wouter van der Wijngaart ${ }^{1}$
}

Whereas electric circuits and surface-based (bio)chemical sensors are mostly constructed in-plane due to ease of manufacturing, 3D microscale and nanoscale structures allow denser integration of electronic components and improved mass transport of the analyte to (bio)chemical sensor surfaces. This work reports the first out-of-plane metallic nanowire formation based on stretching of DNA through a porous membrane. We use rolling circle amplification (RCA) to generate long single-stranded DNA concatemers with one end anchored to the surface. The DNA strands are stretched through the pores in the membrane during liquid removal by forced convection. Because the liquid-air interface movement across the membrane occurs in every pore, DNA stretching across the membrane is highly efficient. The stretched DNA molecules are transformed into trans-membrane gold nanowires through gold nanoparticle hybridization and gold enhancement chemistry. A $50 \mathrm{fM}$ oligonucleotide concentration, a value two orders of magnitude lower than previously reported for flat surface-based nanowire formation, was sufficient for nanowire formation. We observed nanowires in up to $2.7 \%$ of the membrane pores, leading to an across-membrane electrical conductivity reduction from open circuit to $<20 \Omega$. The simple electrical read-out offers a high signal-to-noise ratio and can also be extended for use as a biosensor due to the high specificity and scope for multiplexing offered by RCA.

Keywords: DNA stretching; DNA detection; gold nanowires; nanowire synthesis; membrane

Microsystems \& Nanoengineering (2018) 4, 17084; doi:10.1038/micronano.2017.84; Published online: 12 February 2018

\section{INTRODUCTION}

Conductive nanowire structures are investigated as components in sensors and electronic devices ${ }^{1}$. In sensing applications, nanowires offer many advantages, such as high surface-tovolume ratios, Debye lengths comparable to the target molecule, minimum power consumption, and relatively easy incorporation into microelectronic devices. In electronic engineering, conductive nanowires might offer a solution to the increasing demand for vertical electrical connections (vias) that pass completely through silicon wafers or dies. This process produces high-performance interconnects for creation of three-dimensional (3D) packages and 3D integrated circuits (ICs) ${ }^{2}$ because the density of such vias is substantially higher and the length of the connections is shorter than those manufactured by alternative techniques such as wirebonding and flip-chip processes. Moreover, the combination of electrical conductivity and mechanical flexibility make nanowire conductors attractive for use in flexible electronics ${ }^{3}$.

In recent years, interest has grown in construction of onedimensional metal-enhanced conductive wires from DNA scaffolds. The poor electrical properties of $D N N A^{4}$ can be overcome by building metal nanowires in which the double-stranded DNA acts as a template for the seed-mediated growth of silver ${ }^{5}$, gold ${ }^{6-8}$, or palladium ${ }^{9,10}$ wires. Russell et al. ${ }^{8}$ reported electrical biosensing by generating gold nanoparticle (AuNP)-seeded conductive metal wires that can be electrically sensed, and this approach was enabled by the use of rolling circle amplification (RCA), allowing for the detection of a few nanograms of genomic DNA from Escherichia coli. In all previous methods, metal enhancement occurs either after the fixation of the DNA strand to a flat surface, resulting in in-plane fixated nanowires ${ }^{5,6,8-10}$, or with use of a process in bulk solution ${ }^{7}$.

In the current study, we extend previous work on in-plane nanowire formation to out-of-plane nanowire formation, which can be applied in 3D microelectrical devices for biological sensing applications $^{11-13}$. We demonstrate out-of-plane stretching of DNA amplified by RCA through the pores of a porous membrane, followed by gold enhancement chemistry. We verify the successful and highly efficient nanowire formation using scanning electron microscopy (SEM), confocal microscopy, and electrical conductivity measurements.

\section{MATERIALS AND METHODS}

\section{Gold nanowire preparation}

The process that combines electrode fabrication with the method of stretched rolling circle products (RCPs) and gold nanowires (AuNWs) is shown in Figure 1 and Supporting information S1. The detailed description of the synthesis of DNA AuNWs based on DNA templates is described in this section.

(a) Electrode evaporation. As substrates we used polycarbonate membranes (Whatman, Florham Park, Morris County, New Jersey, USA) with a diameter of $25 \mathrm{~mm}$, a thickness of $10 \mu \mathrm{m}$ and containing straight pores of diameter $2 \mu \mathrm{m}$ and pore surface density of $2.8 \times 10^{6}$ pores per $\mathrm{cm}^{2}$ (SEM in Supplementary Information S2). A $5 \mathrm{~nm} / 100 \mathrm{~nm} \mathrm{Ti/Au} \mathrm{layer} \mathrm{was} \mathrm{deposited} \mathrm{on}$

\footnotetext{
${ }^{1}$ Department of Micro and Nanosystems, KTH Royal Institute of Technology, Osquldas väg 10, Stockholm 10044 , Sweden and ${ }^{2}$ Science for Life Laboratory, Department of Biochemistry and Biophysics, Stockholm University, Tomtebodavägen 23 A, Solna SE-171 65, Sweden

Correspondence: Mats Nilsson or Wouter van der Wijngaart (mats.nilsson@scilifelab.se or wouter@kth.se)

Received: 26 June 2017; revised: 28 August 2017; accepted: 13 September 2017
} 

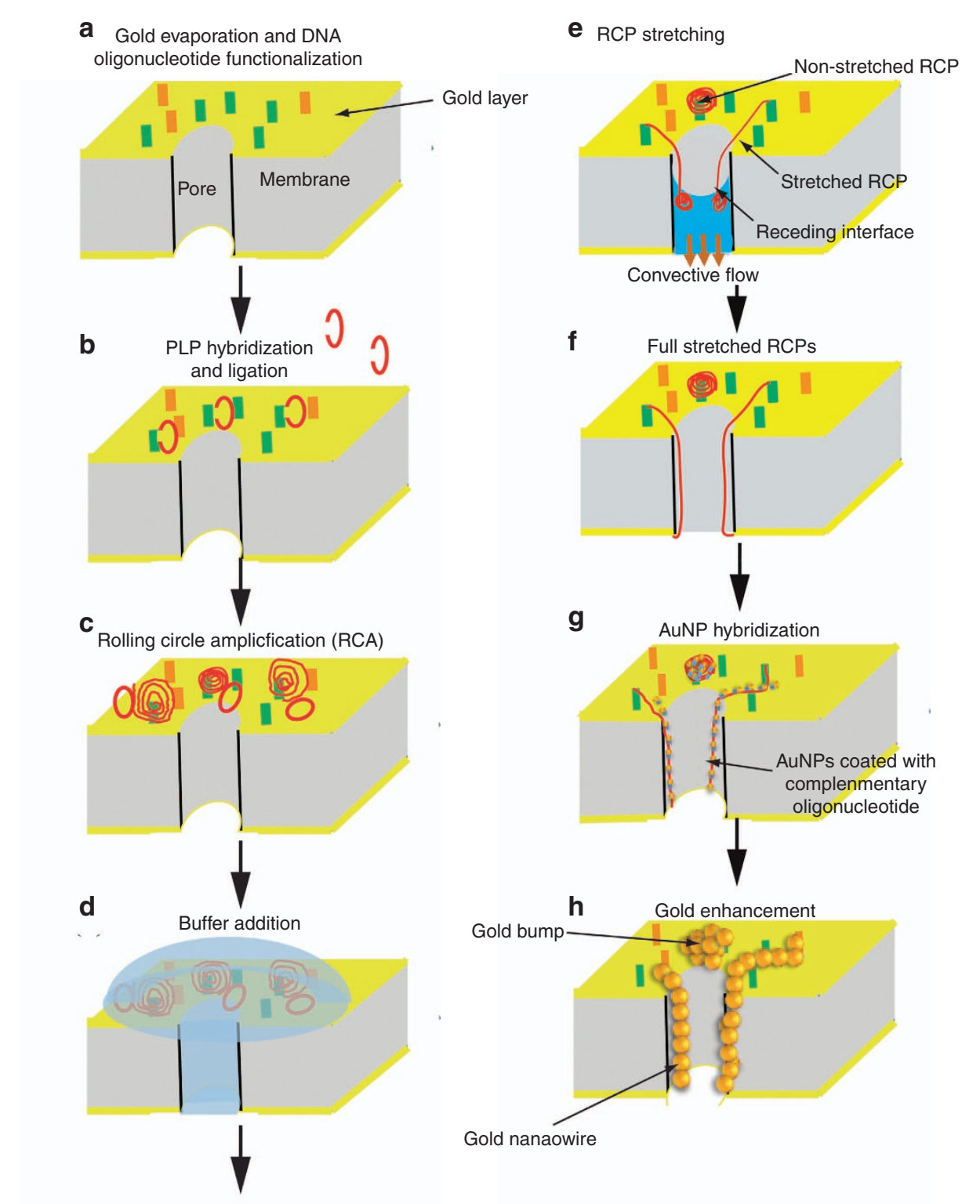

DNA templete

PEG4 backfiller

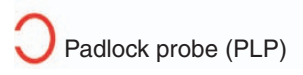

Enhanced AuNP

Figure 1 Schematic cross-sectional illustration of DNA-based synthesis of gold nanowires through a porous membrane. (a) Polycarbonate membranes are coated with $\mathrm{Ti}$ and $\mathrm{Au}$ by evaporation, and the gold surface is functionalized with thiol-functionalized DNA oligonucleotides in the presence of PEG4, which serves to control the oligonucleotide density and surface properties of the gold layer. (b) The padlock probe ends hybridize to the immobilized DNA template, forming a circular structure that is sealed by target directed enzymatic ligation. (c) Ligated circles are amplified by rolling circle amplification (RCA) primed by the DNA template to form a rolling circle product (RCP), a long DNA concatamer that curls up into a nano-ball. (d-f) Drying by forced convection of the liquid through the membrane from top to bottom results in the receding liquid-air interface stretching the RCPs through the pores. (g) DNA oligonucleotides functionalized with gold nanoparticles are hybridized to the stretched RCP and (h) gold enhancement of the AuNPs attached to the stretched RCPs forms out-of-plane nanowires connecting opposite surfaces of the membrane. PEG4, Polyethylene glycol 4; AuNP, gold nanoparticle; PLP, padlock probe; RCA, rolling circle amplification; RCP, rolling circle product.

both sides of the substrate by evaporation through a shadow mask under a $45^{\circ}$ angle using a planetary mechanism to avoid deposition on the entire walls of the pores, thus ensuring a $64 \mathrm{~mm}^{2}$ footprint overlap between the top and bottom electrodes.

Membrane functionalization. The membranes were placed in polystyrene six-well cell culture dishes with a $34.8 \mathrm{~mm}$ diameter (Corning, New York, USA) during all assay steps, except during across-membrane liquid pumping and air drying. First, the membranes were washed using phosphate-buffered saline $(\mathrm{pH} 7.4)$ at room temperature (RT) for $5 \mathrm{~min}$. The membranes were subsequently dried by exposure to vacuum at the membrane bottom. The top gold electrode surface was functionalized with thiolated template oligonucleotides (IDT) with the sequence 5'-SH-TCTCTCTCTCTCT CTCAGTAGGGAGGAAGGTGGTTAAGTTAATA-3'. The oligonucleotide was first activated by incubation at RT for $1 \mathrm{~h}$ in a $20 \mu \mathrm{M}$ solution 
with freshly prepared $100 \mathrm{mM}$ dithiothreitol (DTT; Sigma-Aldrich, St. Louis, MO, USA) in $10 \mathrm{mM}$ phosphate buffer at $\mathrm{pH}$ 8.0. After incubation, the DTT was removed by passing the solution through a NAP-5 column (Sigma, Sigma-Aldrich, St. Louis, MO, USA). The column was washed 5 times with $2 \mathrm{ml}$ of water. Thereafter, $200 \mu \mathrm{L}$ of the oligonucleotide solution was passed through the column, and $300 \mu \mathrm{L}$ of water was added. Elution was conducted by adding $500 \mu \mathrm{L}$ of water. The elute was recovered, and a functionalization mixture containing $10 \mu \mathrm{M}$ activated oligonucleotide, $10 \mu \mathrm{M}$ Polyethylene glycol (PEG) $\mathrm{CH}_{3}$ backfiller (Sigma Sigma-Aldrich, St. Louis, MO, USA), $0.05 \%$ sodium dodecyl sulfate (SDS; Sigma Sigma-Aldrich, St. Louis, MO, USA), and $1 \mathrm{M} \mathrm{NaCl}$ (Sigma-Aldrich, St. Louis, MO, USA) in $10 \mathrm{mM}$ phosphate buffer at $\mathrm{pH} 8.0$ was prepared and added to the membranes followed by incubation at $4{ }^{\circ} \mathrm{C}$ overnight. Finally, the membranes were washed with $0.1 \times \mathrm{SSC}$ buffer and dried by applying vacuum at the membrane bottom.

(b-c) Padlock probe ligation and RCA. The functionalized dried membranes were subjected to padlock probe (PLP) ligation and subsequent RCA. For this process, a $100 \mu \mathrm{L}$ ligation mix containing different concentrations ( $1 \mathrm{nM}, 100 \mathrm{pM}, 100 \mathrm{fM}, 50 \mathrm{fM}$, and $10 \mathrm{fM}$ ) of PLP $\left(5^{\prime}-\mathrm{PO}_{4}\right.$-CTTCCTCCCTACTGAAGAGTGTACCGACCTCTCGTC GAAGTAGCCGTGACTATCGACTTGCGTCTATTAGTGGAGCCTATTAA CTTAACCAC- $3^{\prime}$ ), $0.4 \mu \mathrm{g} \mathrm{L}^{-1}$ BSA (New England Biolabs, Ipswich, MA), $1 \times$ phi29 polymerase buffer $(33 \mathrm{mM}$ Tris-acetate $\mathrm{pH}-7.9)$, $10 \mathrm{mM}$ Mg-acetate, $66 \mathrm{mM}$ K-acetate, 0.1\%(v/v), Tween 20, $1 \mathrm{mM}$ DTT (Thermo Scientific, Waltham, MA, USA), 1 mM ATP (DNA Gdansk Blirt S.A., Poland) and $50 \mathrm{mU} \mathrm{\mu L}^{-1}$ T4 DNA ligase (DNA Gdansk) were added to the membranes and incubated at $37^{\circ} \mathrm{C}$ for 60 min. Thereafter, the specific PLPs are ligated to form circular DNA molecules (Figure 1b) that are subsequently amplified by RCA. The membranes were washed by exchanging the solution for $100 \mu \mathrm{L} 1 \times$ TNT buffer, followed by a second wash with $1 \times$ TNT-0.1\% SDS buffer (wash buffer). The wash buffer was discarded, and an RCA amplification mix containing $0.2 \mu \mathrm{g} \mathrm{L}^{-1}$ BSA, 1X phi29 polymerase buffer, $250 \mathrm{mM}$ dNTPs (DNA Gdansk), and $200 \mathrm{mU} \mathrm{HL}^{-1}$ phi29 polymerase (O-Link Bioscience $A B$, Uppsala, Sweden) was added to the membranes and incubated at $37^{\circ} \mathrm{C}$ for $90 \mathrm{~min}$, followed by a single wash with $0.1 \times$ TNT- $0.1 \%$ SDS buffer to obtain RCPs attached to the membrane (Figure 1c).

(d-f) RCP stretching. RCPs were stretched through the membrane pores by drying under the condition of forced liquid removal through the membrane. This process was performed by placing the wet membrane on a micropore filter and applying vacuum to the bottom during the washing step. During this process, the fixated end of the DNA remains tethered to the top surface, while the free end (which has a lower free enthalpy in the solubilized state) preferably remains in the liquid phase and is transported through the pores to the bottom surface by the wet side of the receding liquid-air interface (Figure 1e).

( $\mathrm{g}-\mathrm{h})$ Gold chemistry and enhancement. For production of DNA AuNWs, a $50 \mathrm{nM}$ aliquot of $10 \mathrm{nM}$ oligonucleotide (complementary to portions of monomeric units of stretched RCPs) functionalized ( $5^{\prime}$-AuNP -TाTाTGCGTCTAGTGGAGCC-3') AuNPs (10 $\mathrm{nM}$ diameter) was used. Hybridization of these AuNPs to stretched RCPs was performed at $37^{\circ} \mathrm{C}$ for $2 \mathrm{~h}$ with shaking in a buffer containing $0.5 \mathrm{M} \mathrm{NaCl}, 20 \mathrm{mM}$ Tris- $\mathrm{HCl}, 20 \mathrm{mM}$ EDTA, and $0.01 \%$ Tween resulting in the RCPs becoming decorated with AuNPs (Figure 1g). After this incubation, the unbound AuNPs were removed using a wash buffer rinse, followed by drying of the membranes using a vacuum pump. The adsorbed AuNPs served as nucleation sites during subsequent gold enhancement at RT with GoldEnhance solution (Nanoprobes Inc. Yaphank, New York, USA) to convert the AuNP-decorated RCPs into AuNWs (Figure 1h)). The time of incubation with this enhancement solution was varied between $10-55$ min (with a step of $5 \mathrm{~min}$ ) at
RT, and the resistance of each sample was measured after rinsing and drying of the membranes.

For the fluorescence assay, step $\mathrm{h}$ was omitted and step $\mathrm{g}$ was altered as follows: RCPs were labelled with a fluorescently tagged oligonucleotide (5'-Cy3-TATTAACTTAACСАCСТTCCAA-3'). This oligo was diluted to $10 \mathrm{nM}$ in $1 \times$ hybridization buffer $(10 \mathrm{mM}$ Tris- $\mathrm{HCl} \mathrm{pH} 7.5,5 \mathrm{mM}$ EDTA, $1 \mathrm{M} \mathrm{NaCl}, 0.1 \%$ Tween 20), and the membranes were incubated at $37^{\circ} \mathrm{C}$ for $2 \mathrm{~h}$ and washed and dried as described above.

\section{Characterization}

Scanning electron microscopy. SEM images of the membrane top surfaces were collected using a Hitachi SEM-Zeiss Ultra 55 instrument (ZEISS, Groupd headquarters, Oberkochen, Germany). An acceleration voltage of $3.5 \mathrm{kV}$ was used during imaging of the membranes.

Confocal laser scanning microscopy. The processed membranes were subjected to confocal fluorescence microscopic analysis. The membrane samples were placed on regular microscope glass slides and covered with $24 \times 24 \mathrm{~mm}^{2}$ cover slips (Menzel, ThermoFisher) after addition of SlowFade Gold Antifade reagent (ThermoFisher) to prevent bleaching of the fluorophore attached to the oligonucleotides complementary to the stretched RCPs. Confocal images were acquired with an fv1000 instrument (Olympus, Center Valley, Pennsylvania, USA) equipped with a $60 \times$ water immersion objective with a 1.2 numerical aperture. $Z$-sections with a focal depth of $10 \mu \mathrm{m}$ were obtained using a $559 \mathrm{~nm}$ excitation laser and a $567 \mathrm{~nm}$ emission filter compatible with Cy3 spectra. The obtained images were analyzed using Icy (Quantitative Image Analysis Unit, Institute Pasteur, Paris, France).

AuNW surface density measurements. The RCP surface density in the SEM images was calculated from the amount of wire features that terminate at a pore in eight SEM images of randomly chosen electrode sections with area $=6.2 \times 10 \mu \mathrm{m}^{2}$. The RCP surface density in the confocal laser scanning microscopy (CLSM) image is the amount of RCPs visible by confocal microscopy (fv1000, Olympus, USA) divided by the scanned area of $60 \times 60 \mu \mathrm{m}^{2}$, which is equal to $4.4 \mathrm{e}^{5} \mathrm{RCPs} \mathrm{cm}{ }^{-2}$. For both imaging and nanowire density calculations, from $50 \mathrm{fM}$ to $1 \mathrm{nM}$, each membrane was imaged at eight randomly chosen positions with the same scanned surface area, except for the $10 \mathrm{fM}$ PLP concentration, for which the density was measured at only two different positions.

Electrical measurements. Deposition of gold on both sides of the membrane prior to thiol oligonucleotide functionalization allows measurement of the electrical resistance over the membrane. The electrical readout was performed with a four-point probe using the 4200-SCS instrument (Semiconductor Characterisation System) (KEITHLEY/A Tektronix Company, Beaverton, Oregon, USA). The resistance was determined by applying a current in the range of $0-5 \mu \mathrm{A}$ with a $0.5-\mu \mathrm{A}$ step increase while measuring the potential over the membrane. We deliberately kept the feed current low to prevent burning of the AuNWs through resistive heating. However, the low current also limits the precision of the measured resistance, resulting in a minimum detectable resistance of approximately $20 \Omega$.

\section{RESULTS}

AuNW formation was investigated using SEM imaging, confocal microscopy, and electrical resistance measurements between the top and bottom membrane surface. A summary of results is presented in Table 1, and details are given in the following sections. 
Table 1 Summary of measurements of nanoscale gold features for different PLP concentrations

\begin{tabular}{|c|c|c|c|c|c|c|}
\hline Padlock probe concentration & $1 \mathrm{nM}$ & $100 \mathrm{pM}$ & $100 \mathrm{fM}$ & $50 \mathrm{fM}$ & $10 \mathrm{fM}$ & $0 \mathrm{fM}(=\mathrm{ctrl})$ \\
\hline Gold bump density in SEM image \pm SD $\left(10^{7} \mathrm{~cm}^{-2}\right)$ & $4.6 \pm 3.5$ & $0.55 \pm 0.3$ & $5.4 \pm 4.5$ & $6.8 \pm 3.6$ & $3.1 \pm 2.4$ & 0 \\
\hline Gold bump diameter in SEM image \pm SD $(\mathrm{nm})$ & $294 \pm 19$ & $254 \pm 18$ & $267 \pm 23$ & $271 \pm 15$ & $253.5 \pm 8.5$ & - \\
\hline Full AuNW density in SEM image \pm SD $\left(10^{4} \mathrm{~cm}^{-2}\right)$ & $7.5 \pm 3.0$ & $4.2 \pm 2.2$ & $3.2 \pm 1.1$ & $2.2 \pm 0.8$ & 0 & 0 \\
\hline Streched RCP density in CLSM image \pm SD $\left(10^{4} \mathrm{~cm}^{-2}\right)$ & 44 & - & - & - & - & 0 \\
\hline Gold enhancement time needed to reach $R<10^{12} \Omega$ in electrical & $0-35$ & $20-35$ & $10-30$ & 30-35 & $\infty^{+}$ & $\infty^{+}$ \\
\hline
\end{tabular}

characterization ( $\mathrm{min})$

Abbreviations: CLSM, confocal laser scanning microscopy; RCP, rolling circle product; SEM, scanning electron microscope; PLP, padlock probe; AuNW, gold nanowire. ${ }^{a} \infty$ indicates no measurable resistance was detected within 55 min of gold enhancement.
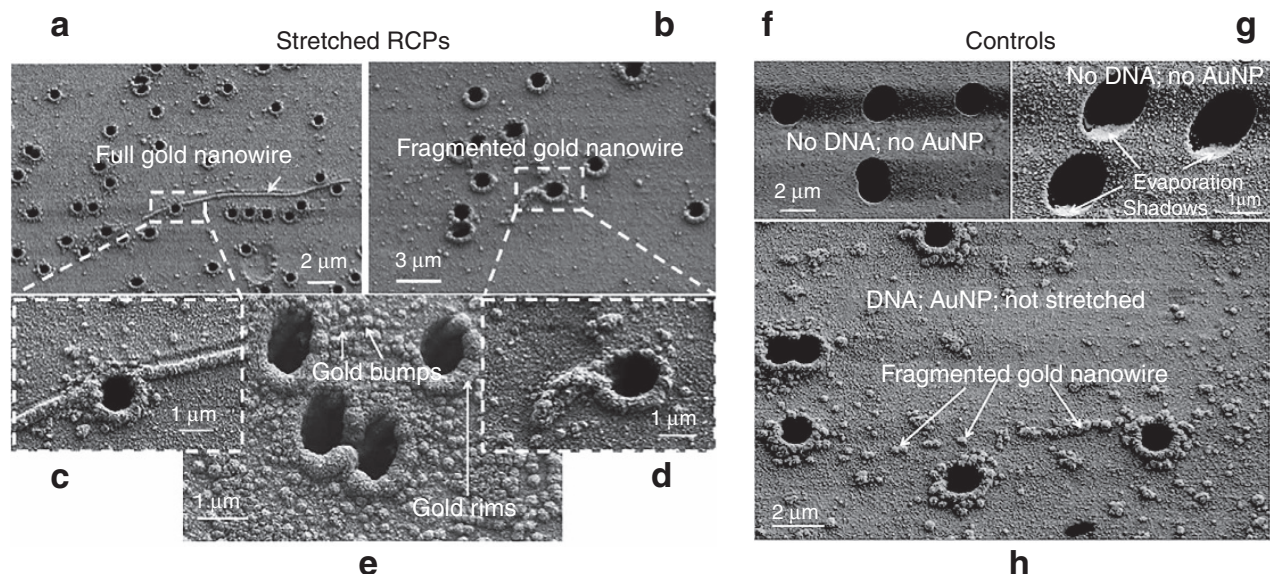

Figure 2 SEM images of the membrane top surface after DNA stretching and 55 min gold enhancement. (a-d) After positive assay; (f-h) after control measurements in which the following steps were omitted from the assay, respectively, (f) both PLP and AuNPs incubation; (g) AuNP incubation; and (h) the DNA stretching step. (a) and (c) full AuNWs are visible on the gold surface; (b) three full AuNWs are stretched towards two pores. AuNP, gold nanoparticle; PLP, padlock probe; SEM, scanning electron microscope; AuNWs, gold Nanowires; RCPs, rolling circle products.

\section{SEM characterization of synthesized nanowires}

To study the AuNW formation, we captured SEM images of the top membrane surface after 55 min of gold enhancement (Figure 2). For assays with RCP stretching with a PLP concentration of $\geqslant 50 \mathrm{fM}$ (Figures 2a-e), we observed the following distinct features on the membrane surface: gold bumps with approximately spherical geometry, fragmented AuNWs (which are strings of gold bumps that stretch over the surface), full AuNWs (hereafter simply referred to as 'nanowires') that stretch over the surface toward the pores with varying length ranging from 3 to $60 \mu \mathrm{m}$, and gold rim structures around all pore openings.

For the assay with PLP concentration $=10 \mathrm{fM}$ and for assays without RCP stretching (Figure $2 \mathrm{~h}$ ), that is, where drying of the RCP products occurred under natural convection instead of forced convection, we observed similar gold bumps, fragmented AuNWs, and gold rims around the pores but not full nanowires. For control assays with zero PLP concentration but with AuNP decoration and gold enhancement (Figures $2 f$ and $g$ ), we did not observe any gold enhancement features. The size and areal distribution of the gold bumps and the full AuNWs are plotted in Figure 3.

\section{Confocal microscopy characterization of the trans-membrane DNA stretching}

To study the spreading of the stretched RCPs inside the pores, we performed CLSM analysis after an assay that began with $1 \mathrm{nM}$ PLP in which the RCPs were labeled with fluorescent detector probes instead of AuNPs. Figure 4 shows CLSM images after an assay with RCP stretching (a) and after an assay without RCP stretching (b) during membrane drying. For the assay with stretching, DNA nanowires of length $10 \pm 1.4 \mu \mathrm{m}$ can be clearly discerned in the membrane through its $z$ axis. The areal density of the stretched RCPs was $4.4 \times 10^{5}$ RCPs per $\mathrm{cm}^{2}$. For the assay without stretching, a fluorescent signal was observed from a ring around the pore openings on the top of the membrane but not inside the pores. Supplementary Information S3 provides the two movies: the positive $3 \mathrm{D}$ result made from the assay with RCP stretching and the negative $3 D$ result formed by the assay without $R C P$ stretching.

\section{Electrical characterization of AuNWs}

We measured the electrical resistance between the top and the bottom of the membrane. For all assays, the membrane constituted an electrically open circuit prior to gold enhancement. Table 1 lists the gold enhancement time needed to reach a detectable electrical resistance $R<10^{12} \Omega$, which is the higher limit of detection (LoD) for the electrical resistance measurement set-up, for different PLP starting concentrations. For all assays with PLP concentrations $\geqslant 50 \mathrm{fM}$, the resistance dropped to a measurable level within $35 \mathrm{~min}$ of gold enhancement. For the assay with $10 \mathrm{fM}$ PLP and for all control assays, the membrane remained an open circuit until the maximum tested 60 min of gold enhancement (I-V curve in Supplementary Information S4).

Figure $5 a$ shows the measured resistance after subsequent 5min intervals of gold enhancement during assays starting from the $50 \mathrm{fM}$ and 100 pM PLP concentrations. Between 20 and $45 \mathrm{~min}$ of gold enhancement, both resistance values drop from an open 


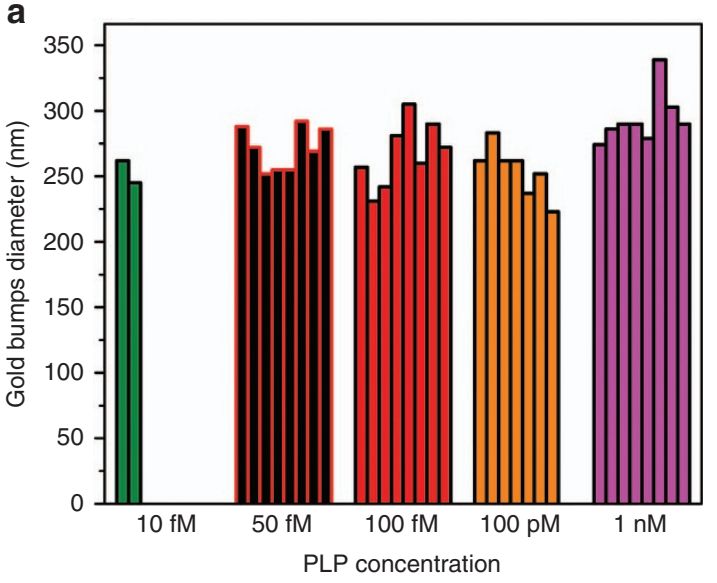

C

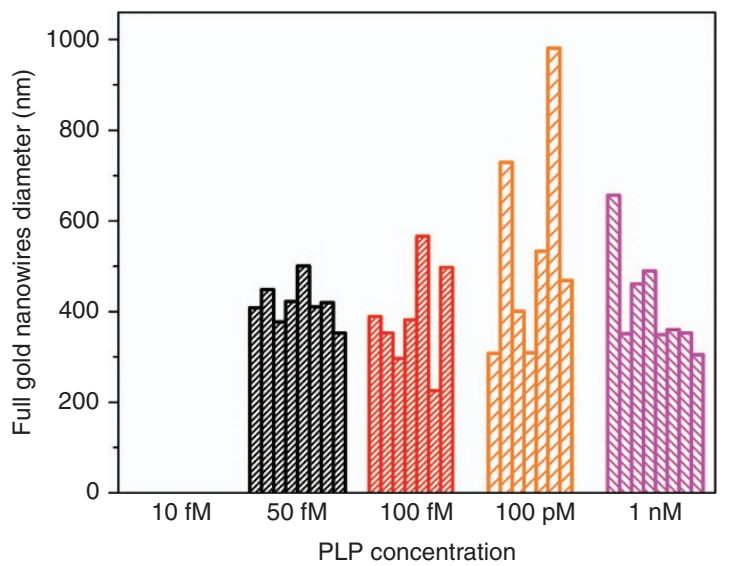

b

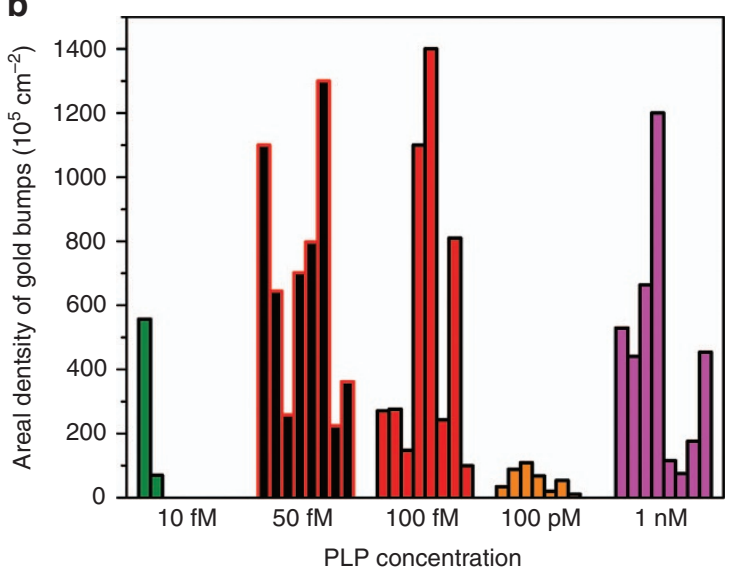

d

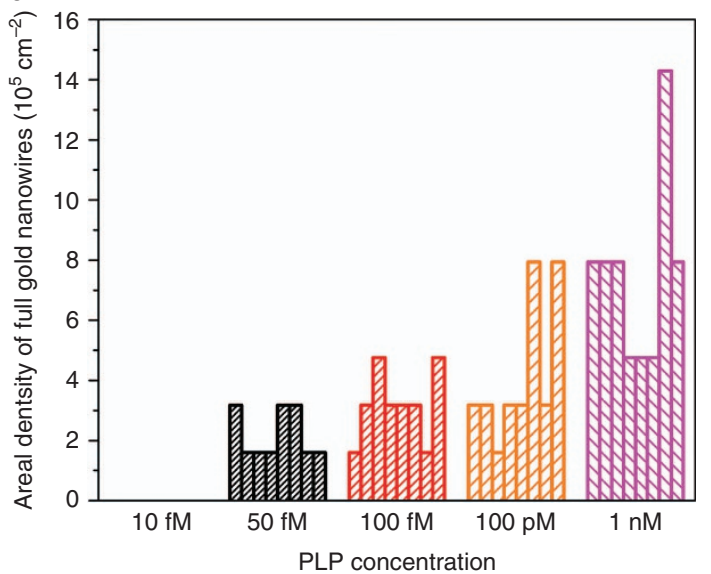

Figure 3 Measured size and areal density of gold bumps ( $\mathbf{a}$ and $\mathbf{b}$ ) and full AuNWs (c and $\mathbf{d}$ ) the membrane surface for different PLP concentrations. Each bar represents an individual measurement of the surface area density on randomly chosen SEM images of the membrane. PLP, padlock probe; SEM, scanning electron microscope; AuNWs, gold nanowires.

circuit to below the lower LoD of the resistance measurement set-up, which was $20 \Omega$. The membranes were divided into regions of $16 \mathrm{~mm}^{2}$ for which the electrical resistance was measured. Figure $5 \mathrm{~b}$ shows the fraction of those regions that are electrically connected after different gold enhancement times for assays starting from different PLP concentrations.

\section{DISCUSSION}

We obtained successful DNA-assisted synthesis of AuNWs through the porous membrane, as evident from the SEM, CLSM, and electrical characterization. The SEM images confirm the presence of the full AuNWs on the top of the membrane and at the entrance of the pores, the CLSM images indicate the stretching of the DNA of the membrane from top to bottom, and the resistance measurements confirm the formation of electrical contacts between the top and bottom of the membranes. Furthermore, all three characterization methods confirm that trans-membrane AuNW formation is selective for assays with RCA followed by active stretching.

Because the liquid-air interface movement across the membrane occurs in every pore, DNA stretching across the membrane is highly efficient. Indeed, the successful generation of DNAassisted full AuNWs from a PLP concentration as low as $50 \mathrm{fM}$ constitutes a method that is more than two orders of magnitude more efficient than that reported in previous DNA stretching on flat surfaces ${ }^{8}$, which required a minimum of 6 pM PLP concentration. The AuNW pore occupancy, defined as the ratio of full AuNW areal density to the pore density, is between 0.8 and $2.7 \%$ for assays starting from $50 \mathrm{fM}$ to $1 \mathrm{nM}$ PLP concentration. We observe only a small correlation between PLP concentration and full AuNW areal density, unlike any noticeable correlation for the PLP concentration with the gold bump density (Figures $3 b$ and $d$, respectively). The fluorescently labeled stretched RCP pore occupancy for assays starting from $1 \mathrm{nM}$ PLP is $16 \%$, that is, one order of magnitude larger than that of the AuNWs observed in the SEM images. This observation might indicate that only a fraction of the AuNWs have a portion of the wire on the top surface of the membrane.

Gold bump formation and fragmented AuNW formation are only observed after assays that include RCA. Single gold bump formation is attributed to non-stretched RCP spheres bonded to the membrane top surface before enhancement by gold chemistry. Because the related events of RCP formation and subsequent AuNW synthesis (which eventually determine the stretching) are directly correlated with the increasing PLP concentration, this trend becomes visible with the AuNW areal density but not in the case of gold bump areal density. Previous observations of non-stretched RCPs after similar RCA assays in solution indicate that RCPs curl up into spherical structures with 


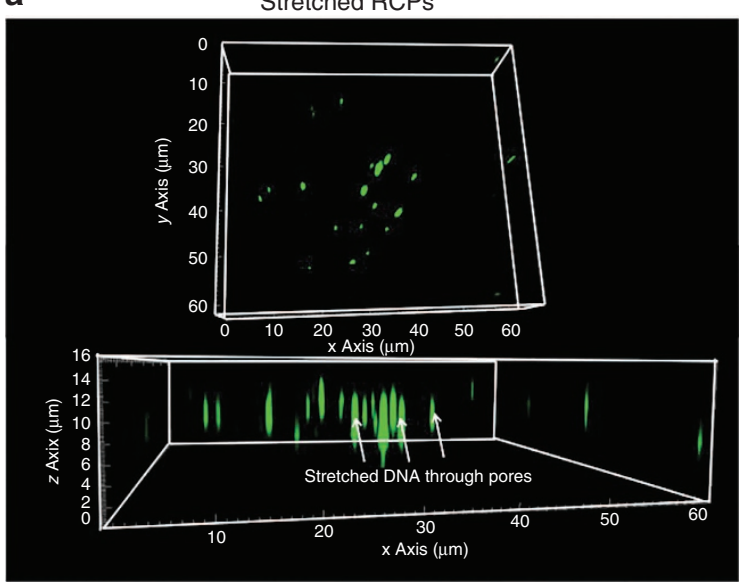

b

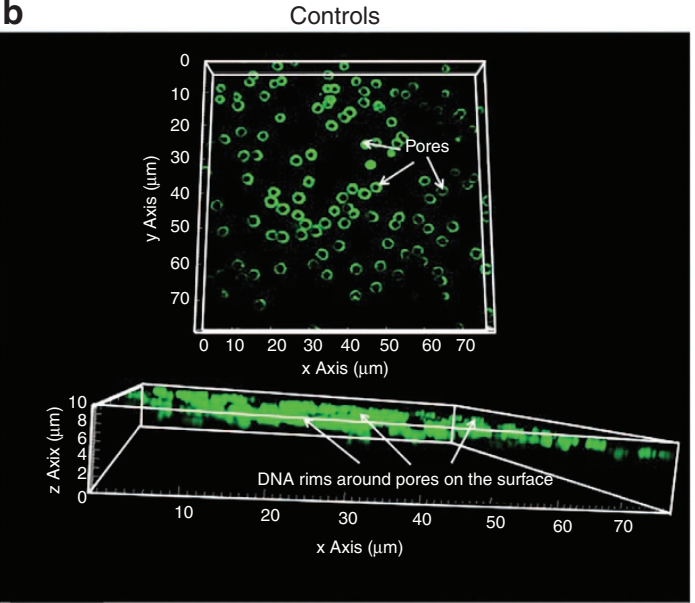

Figure 4 CLSM images of stretched RCPs labeled with fluorescently tagged oligonucleotides. Top view (top) and cross-sectional view (bottom) of 3D confocal images of the membranes after assay with RCP stretching, that is, with forced convection through the membrane by drying (a), and after assay without RCP stretching, that is, without forced convection by drying (b). CLSM, confocal laser scanning microscopy; RCP, rolling circle product; 3D, three dimensional.
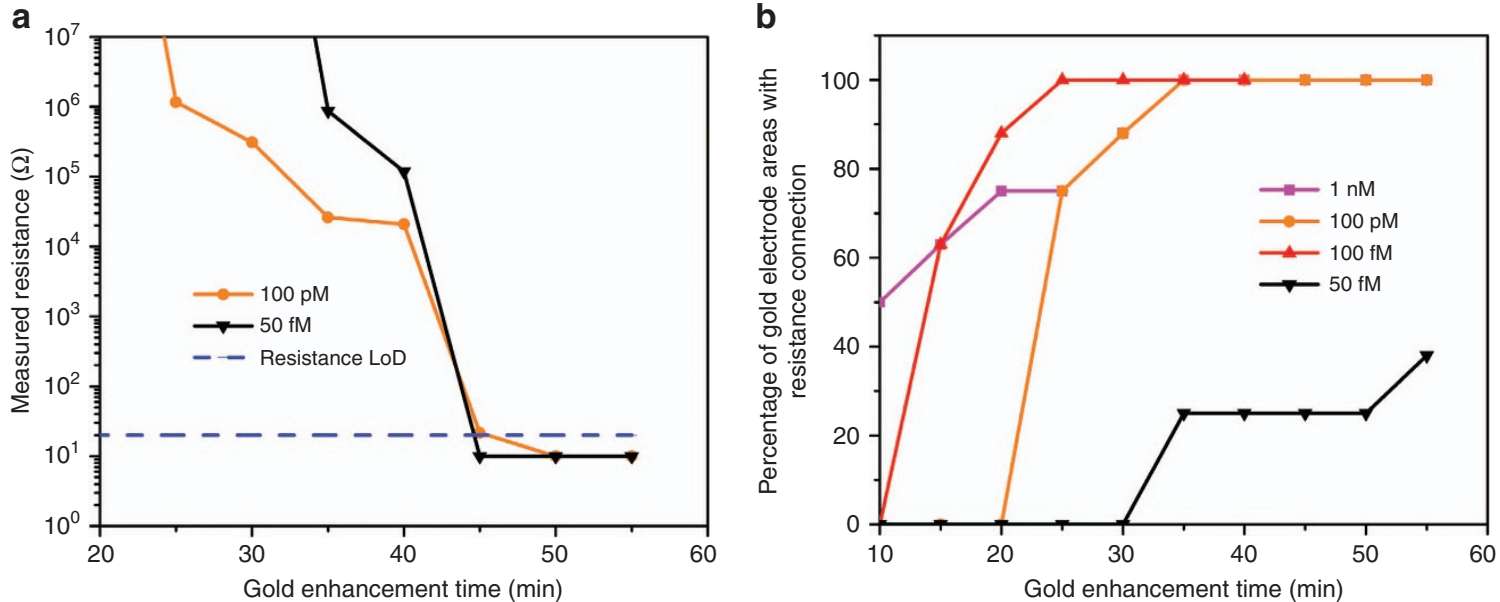

Figure 5 (a) Measured resistance between top and bottom electrode vs. gold enhancement time for $50 \mathrm{fM}$ and $100 \mathrm{pM}$ PLP sample (lines are supplied for eye guidance only). The lower LoD of $20 \Omega$ is indicated with a dashed line. Resistance values above the higher LoD of $10^{12} \Omega$ are not plotted, and resistance values below the lower LoD are plotted as $10 \Omega$. (b) Fraction of the $16 \mathrm{~mm}^{2}$ membrane sections that are electrically connected after different gold enhancement times for PLP samples with concentration $\geqslant 50 \mathrm{fM}$ (lines are supplied for eye guidance only). LoD, limit of detection; PLP, padlock probe.

a typical diameter of $700 \mathrm{~nm}^{14}$. We expect that such spherical RCP structures would shrink when dried on the surface, resulting in bumps of the size observed in Figure 2 after AuNP decoration and gold enhancement. Moreover, a high possibility also exists that the RCPs become attached only partially to a surface that is not completely dried by the vacuum process, and upon subsequent addition of a buffer with high salt content, they undergo re-curling, thus reducing the potential stretching yield (Table 1).

The areal density of gold bumps is three orders of magnitude larger than that of full AuNWs. This result indicates that approximately only one in a thousand RCPs bonding to the gold surface is stretched through a pore. Despite the significant improvement in AuNW synthesis compared with previous work by Russel et $a l^{8}{ }^{8}$, the RCP stretching still leaves ample room for improvement. The stretching of an RCP requires a receding solidliquid-air triple interface line that leaves one end of the RCP tethered to the dry surface and the other end remaining in solution, with the latter thermodynamically more favorable. We hypothesize that, during drying of the membrane, small pools of liquid remain on the membrane surface between the pores, where they dry and thereby concentrate the RCPs. Indeed, areas of high and low gold bump areal concentration can be distinguished in the SEM images in Supplementary Information S5 and S6. The effect is also reflected in the large spread in gold bump areal density in Figure 3. The difference between the gold bump and full AuNW densities indicates that an improved stretching method could further decrease the minimum PLP concentration by three orders of magnitude.

Fragmented AuNW formation is attributed to stretching of RCPs after AuNP decoration but before Au enhancement chemistry. We hypothesize that, for unstretched RCPs, steric hindrance limits the amount of AuNPs that can bind to the RCP. If these RCPs are stretched during drying prior to gold enhancement chemistry, 
only the specific regions of the RCP that captured AuNWs are expected to become enhanced and form bumps.

Gold rim formation visible in the SEM images and fluorescent ring formation around the pore openings visible in the CLSM images are attributed to capillary forces that retain liquid in the pores as the last portions to dry, leading to an increasing concentration of the RCPs in and around the pores. This local increase in RCP concentration thermodynamically favors bonding of the RCP to the surface probes on the gold surface immediately surrounding the pore openings. The resulting high RCP surface concentration results in the formation of gold rims or fluorescent rings after AuNP decoration, gold enhancement, and fluorescent probe decoration.

The trans-membrane AuNWs constitute high areal density and high aspect ratio conductors, with electrical contact to both the top and the bottom of the membrane. Patterning the gold layer on the membrane top and bottom surface in small isolated islands prior to performing the assay results in membranes with anisotropic vertical conductance. Such geometries might find applications as interposer layers in IC stacking or as contact layers between electronic dies and sensor layers.

The novel method has potential for use in future biosensing and electronic applications. Our novel approach holds promise for future applications in multiplexed point-of-care sensing of lowabundance biomolecules in a wide range of clinical, military, and environmental monitoring settings based on the following key features: the generation and stretching of RCPs that form transmembrane nanowires is simple and rapid; electrical conductivity detection supplies a high signal-to-noise ratio; RCA offers a high specificity; the membrane geometry is compatible with enhanced multiplexing and validation, without any compromise in simplicity and sensitivity; the biosensor can be adapted for different types of biomolecular sensing, including nucleic acids and proteins, when combined with proximity ligation; and an appropriate choice of the sensing area combined with the potential use of multiple electrodes allows a large dynamic range. From the sensor point of view, a more stringent optimization of geometrical design parameters could be considered for enhanced device performance. In its current form, the method can be applied in sensing the presence of PLP with a concentration of $\geqslant 50 \mathrm{fM}$. The ratio between non-stretched and stretched DNA is three orders of magnitude, and hence, improved DNA stretching might allow a thousand-fold increase in the LoD. Specifically, decreasing the distance between pores is expected to improve the likelihood for the RCP stretching rather than remaining curled up on the membrane surface between the pores. The sensor format is well suited for miniaturized realization using silicon-based manufacturing with standard Micro-electromechanical Systems (MEMS) and integrated circuit (IC) manufacturing tools. Such a realization paves the way for performance-oriented device design such as geometrical optimization of membrane thickness, pore size and pore pitch, and spatially separated miniaturized multiplexed electrode areas to allow for digital bioassays and on-chip integration of electrical read-out and data processing.

From the bioassay point of view, the assay can be tuned for multiplexed analyte detection by multiplexed oligonucleotide functionalization and a combinatorial protein read-out system by adopting the proximity ligation assay ${ }^{15}$. The possible improvements, such as spatially separated gold electrodes functionalized with relevant pathogen/biomarker-specific probes, can extend the current device application as a powerful diagnostic tool in clinical settings, for example, for the detection of different pathogens ${ }^{16}$, antibiotic resistance markers ${ }^{17}$, cancer mutations, and in situ transcriptome analysis ${ }^{18}$.

\section{CONCLUSIONS}

We have demonstrated an AuNW-based electrical detection of amplified DNA molecules on a porous membrane substrate. Template-specific long-ssDNA concatemers were generated by RCA and stretched using forced drying through the membrane pores. DNA AuNWs were drawn from these RCPs by hybridizing oligo-functionalized AuNPs along the entire length of the stretched DNA, followed by a gold enhancement protocol for metallization. The resulting enhancement in the conductivity was read as a decrease in across-membrane electrical resistance. The topographical and out-of-plane features of the DNA nanowire were studied using methods such as SEM and CLSM. From the electrical measurements, we confirmed that the resistance across the membrane changed from an open circuit value to below $20 \Omega$ within $45 \mathrm{~min}$. For the current device set-up, we have shown that concentrations of PLP as low as $50 \mathrm{fM}$ become electrically detectable via our novel approach. Use of this novel approach for biosensing holds great promise for future applications in multiplexed point-of-care sensing of low-abundance biomolecules in a wide range of military and environmental monitoring settings and in clinical applications such as multiplexed biomarker detection in infectious disease and cancer diagnosis.

\section{ACKNOWLEDGEMENTS}

We acknowledge support from the European Union's Horizon 2020 research and innovation programme ND4ID under the Marie Sklodowska-Curie grant agreement No. 675412. We also acknowledge the Swedish Research Council (VR) and Swedish Foundation for Strategic Research (SSF) grant (Flu-ID project No. SBE13-0125). MG acknowledges financial support from the China Scholarship Council (CSC) in China. We also express gratitude to Cecilia Aronsson for assistance with the sample preparation.

\section{COMPETING INTERESTS}

The authors declare no conflict of interest.

\section{REFERENCES}

1 Ramgir NS, Yang Y, Zacharias M. Nanowire-based sensors. Small 2010; 6: 1705-1722.

2 Graef $M$, Huizing B, Mahnkopf $R$ et al., International Technology Roadmap for Semiconductors 2.0 (ITRS). Chapter 62015

3 Liu Z, Xu J, Shen G et al. Flexible electronics based on inorganic nanowires. Chemical Society Reviews 2015; 44: 161-192.

4 Welch K, Blom T, Stromme M et al. Enabling measurements of low-conductance single molecules using gold nanoelectrodes. Nanotechnology 2011; 22: 125707.

5 Braun E, Eichen Y, Ben-Yoseph G et al. DNA-templated assembly and electrode attachment of a conducting silver wire. Nature 1998; 391: 775-778.

6 Braun E, Keren K. From DNA to transistors. Advances in Physics 2004; 53: 441-496.

7 Kundu S, Maheshwari V, Saraf RF. Photolytic metallization of Au nanoclusters and electrically conducting micrometer long nanostructures on a DNA scaffold. Langmuir 2008; 24: 9668-9674.

8 Russell C, Welch K, Nilsson M et al. Gold nanowire based electrical DNA detection using rolling circle amplification. ACS Nano 2014; 8: 1147-1153.

9 Nguyen K, Monteverde M, Bourgoin JP et al. Synthesis of thin and highly conductive DNA-based palladium nanowires advanced materials. Advanced Materials 2008; 20: 1099-1104.

10 Richter J, Seidel R, Schackert HK et al. Nanoscale palladium metallization of DNA. Nanoscale 2000; 94: 8720.

11 Galland R, Leduc P, Guérin C et al. Fabrication of three-dimensional electrical connections by means of directed actin self-organization. Nature Materials 2013; vol. 12: 416-421.

12 Shi L, Chu Z, Liu Y et al. Facile fabrication of a three-dimensional gold nanowire array for high-performance electrochemical sensing. Journal of Materials Chemistry B 2015; 3: 3134-3140.

13 Rahong S, Yasui T, Yanagida $T$ et al. Three-dimensional nanowire structures for ultra-fast separation of DNA, protein and RNA molecules. Scientific Reports 2015; 5: 10584.

14 Melin J, Jarvius J, Nilsson $M$ et al. Homogeneous amplified single-molecule detection: Characterization of key parameters. Analytical Biochemistry 2007; 368: 230-238. 
15 Fredriksson S, Gullberg M, Landegren $U$ et al. Protein detection using proximitydependent DNA ligation assays. Nature Biotechnology 2002; 20: 473-477.

16 Göransson J, Ke R, Nilsson M et al. Rapid identification of bio-molecules applied for detection of biosecurity agents using rolling circle amplification. PLOS ONE 2012; 7: e31068.

17 Mezger A, Gullberg E, Andersson DI et al. A General method for rapid determination of antibiotic susceptibility and species in bacterial infections. Journal of Clinical Microbiology 2015; 53: 425-432.

18 Ke R, Mignardi M, Nilsson $M$ et al. In situ sequencing for RNA analysis in preserved tissue and cells. Nature Methods 2013; 10: 857-860. cc) (i) This work is licensed under a Creative Commons Attribution 4.0 International License. The images or other third party material in this article are included in the article's Creative Commons license, unless indicated otherwise in the credit line; if the material is not included under the Creative Commons license, users will need to obtain permission from the license holder to reproduce the material. To view a copy of this license, visit http://creativecommons.org/licenses/ by/4.0/

(c) The Author(s) 2018

Supplementary Information for this article can be found on the Microsystems \& Nanoengineering website (http://www.nature.com/ micronano). 